\title{
A Dielectric Instability of a Fermion Gas in a Random Potential
}

\author{
V. Dallacasa \\ Istituto di Fisica dell'Università, Via Massimo d'Azeglio 85, 43100 Parma \\ Gruppo Nazionale Struttura della Materia CNR - Italy
}

(Z. Naturforsch. 32 a, 844-853 [1977]; received June 6, 1977)

\begin{abstract}
A dielectric instability of a collection of charged fermions interacting via Coulomb forces and subjected to a random external field is pointed out. It is shown that, as a result of localization produced by the random potential, an RPA Coulomb field can generate a dielectric instability, both static or frequency dependent, resulting in a static or oscillating crystalline arrangement of the fermions. In simple metals, such as the alkali metals, this mechanism is shown to provide an explanation of the existence of the crystal lattice and of acoustic phonons.
\end{abstract}

\section{Introduction}

Since Anderson's work in $1958^{1}$ a growing interest has been devoted to solutions of the Schrödinger equation with a random potential. It is now commonly accepted that solutions exist corresponding to localized states, filling a continuous range of energies, along with extended states similar to planewave-like solutions: the envelopes of such states fall off exponentially from some point in space and, in the absence of any external disturbance, are conveniently thought of as distributed uniformly in space $^{2}$.

If charged particles are supposed to fill these states, it becomes important to take the Coulomb interaction into account which is expected to produce much larger effects than on charges occupying extended states ${ }^{2}$.

This aspect is relevant in experimental situations such as the conductance of amorphous semiconductors at sufficiently low temperature, where a hopping mechanism at localized states becomes dominant, so that the inclusion of correlation is necessary to get a complete theoretical model ${ }^{3,4}$. However the problem appears to be much broader than such experiments would suggest, and other situations can be devised where the localization of charges under the influence of the coulomb repulsion plays a decisive role. In this paper a formal treatment of this problem is given for low temperatures, taking the charge interaction into account in a random phase approximation, following closely a standard approach $^{5}$ available for extended, plane-wave like, states. The approach will be that of calculating the

Reprint requests to Istituto di Fisica, Via M. d'Azeglio, 85 I-43100 Parma, Italy. wave vector dependent dielectric constant from which information can be extracted on collective modes which we will find more useful than single particle excitations.

A situation which can be analyzed this way is that of the crystal lattice of the simplest metals, where the nuclei plus cores can be treated as charged identical particles localized by the potential due to the electrons. In the Born-Oppenheimer approximation in fact the electron motion can be treated as much faster than that of the nuclei so that, seen from the nuclei, the electrons generate a complete random potential. The lattice is then the result of a collective static mode, i. e. at zero energy, arising from the vanishing of the static dielectric function, and the lattice vibrations can be viewed as dynamical collective modes which arise, likewise, from the vanishing of the frequency-dependent dielectric function.

In Section 2 a detailed mathematical account will be given of the dielectric function calculation, closely resembling the usual RPA approach ${ }^{5}$, as well as some discussion of a further approximation, besides the RPA, necessary to deal with localized states. In Section 3 the lowest order result in the random potential are worked out, corresponding to treat the random potential as a spatial average, and the way in which the zero of the dielectric function is obtained is discussed. The simplest metals, such as the alkali metals are shown to belong to this case in Section 4, by working out explicitly the BornOppenheimer approximation leading to the random potential point of view. The wavevector of the static mode leads to an explicit expression for the lattice spacing, in fair agreement with measured values, while the group velocity of the dynamical mode coincides with the sound velocity in the acustic branch of the phonon spectrum. 


\section{The Dielectric Constant for Localized States}

The calculation of the dielectric constant can be carried out in much the same way as the standard approach leading to Lindhard's result ${ }^{5}$. We introduce a local potential $U(\boldsymbol{r})$ into the system we are considering and suppose it so weak that first order terms only must be retained in the response. In particular the variation in charge density induced by $U(\boldsymbol{r})$ can thus be written:

$$
\begin{aligned}
& \Delta \varrho(\boldsymbol{r})=Z e_{-\infty}^{+\infty} \mathrm{d} \varepsilon f(\varepsilon) \operatorname{Im} \int \mathrm{d} \boldsymbol{r}^{\prime \prime} G\left(\boldsymbol{r}, \boldsymbol{r}^{\prime \prime} ; \varepsilon\right) \\
& \cdot U\left(\boldsymbol{r}^{\prime \prime}\right) G\left(\boldsymbol{r}^{\prime \prime}, \boldsymbol{r} ; \varepsilon\right)
\end{aligned}
$$

where $Z e$ is the charge of each particle in the system, $f(\varepsilon)$ is the energy distribution function, which from now on we shall take as the Fermi Dirac distribution appropriate to fermions for reasons to be discussed later, and $G\left(\boldsymbol{r}, \boldsymbol{r}^{\prime \prime} ; \varepsilon\right)$ is the double time Green function ${ }^{6}$ :

$$
G\left(\boldsymbol{r}, \boldsymbol{r}^{\prime \prime} ; \varepsilon\right)=\left\langle\psi(\boldsymbol{r}) ; \psi^{+}\left(\boldsymbol{r}^{\prime \prime}\right)\right\rangle_{\varepsilon}
$$

according to Zubarev's scheme, $\psi(\boldsymbol{r})$ and $\psi^{+}(\boldsymbol{r})$ being the destruction and creation operators for a particle at a point $\boldsymbol{r}$.

From (2.1) the self-consistent field $\Delta \Phi(\boldsymbol{r})$ follows, as generated by the particle interaction:

$$
\begin{aligned}
\Delta \Phi(\boldsymbol{r})= & 4 \pi Z^{2} e^{2} \int_{-\infty}^{+\infty} \mathrm{d} \varepsilon f(\varepsilon) \operatorname{Im} \int \mathrm{d} \boldsymbol{r}^{\prime \prime} \int \mathrm{d} \boldsymbol{r}^{\prime} \\
& \cdot \frac{G\left(\boldsymbol{r}^{\prime}, \boldsymbol{r}^{\prime \prime} ; \varepsilon\right) U\left(\boldsymbol{r}^{\prime \prime}\right) G\left(\boldsymbol{r}^{\prime \prime}, \boldsymbol{r}^{\prime} ; \varepsilon\right)}{\left|\boldsymbol{r}-\boldsymbol{r}^{\prime}\right|}
\end{aligned}
$$

which is to be included in $U(\boldsymbol{r})$ in a self-consistent way, i. e.:

$$
U(\boldsymbol{r})=U^{(\mathrm{e})}(\boldsymbol{r})+\int \mathrm{d} \boldsymbol{r}^{\prime \prime} \chi\left(\boldsymbol{r}, \boldsymbol{r}^{\prime \prime}\right) U\left(\boldsymbol{r}^{\prime \prime}\right)
$$

where $U^{(\mathrm{e})}(\boldsymbol{r})$ is the "external" field and $\chi\left(\boldsymbol{r}, \boldsymbol{r}^{\prime \prime}\right)$ a response function given by:

$$
\begin{aligned}
\chi\left(\boldsymbol{r}, \boldsymbol{r}^{\prime \prime}\right)= & 4 \pi Z^{2} e^{2} \int_{-\infty}^{+\infty} \mathrm{d} \varepsilon f(\varepsilon) \operatorname{Im} \int \mathrm{d} \boldsymbol{r}^{\prime} \\
& \frac{G\left(\boldsymbol{r}^{\prime}, \boldsymbol{r}^{\prime \prime} ; \varepsilon\right) G\left(\boldsymbol{r}^{\prime \prime}, \boldsymbol{r}^{\prime} ; \varepsilon\right)}{\left|\boldsymbol{r}-\boldsymbol{r}^{\prime}\right|}
\end{aligned}
$$

In general $\chi\left(\boldsymbol{r}, \boldsymbol{r}^{\prime \prime}\right)$ will be a function of $\boldsymbol{r}$ and $\boldsymbol{r}^{\prime \prime}$ separately unless the translational invariance is restored, which in our case can be done by taking the ensemble average of (2.5), according to the random nature of the potential. This is not $U(\boldsymbol{r})$, of course, but a potential which is supposed to be applied to be system when $U(\boldsymbol{r})=0$, along with the
Coulomb interaction between charges. Introducing the notation $\tilde{A}=\langle A\rangle$ as the ensemble average of any quantity $A$, where

$$
\langle A\rangle=\int \mathrm{d}\left\{R_{i}\right\} P\left(\left\{R_{i}\right\}\right) A\left(\left\{R_{i}\right\}\right),
$$

$\left\{R_{i}\right\}$ being a set of random variables and $P\left(\left\{R_{i}\right\}\right)$, their probability density, such as illustrated in Section 3 , we find from (2.5):

$$
\begin{aligned}
\tilde{\chi}\left(\boldsymbol{r}-\boldsymbol{r}^{\prime \prime}\right)= & 4 \pi Z^{2} e^{2} \int_{-\infty}^{+\infty} \mathrm{d} \varepsilon f(\varepsilon) \operatorname{Im} \int \mathrm{d} \boldsymbol{r}^{\prime} \\
& \frac{\left\langle G\left(\boldsymbol{r}^{\prime}, \boldsymbol{r}^{\prime \prime} ; \varepsilon\right) G\left(\boldsymbol{r}^{\prime \prime}, \boldsymbol{r}^{\prime} ; \varepsilon\right)\right\rangle}{\left|\boldsymbol{r}-\boldsymbol{r}^{\prime}\right|}
\end{aligned}
$$

where now $\tilde{\chi}\left(\boldsymbol{r}-\boldsymbol{r}^{\prime \prime}\right)$ depends only on the coordinate differences, so that Eq. (2.4) can be Fourier transformed to give, after taking the ensemble average:

with

$$
\langle U(\boldsymbol{q})\rangle=U^{(\mathrm{e})}(\boldsymbol{q}) / \mathcal{E}(\boldsymbol{q})
$$

and

$$
\varepsilon(\boldsymbol{q})=1-\tilde{\chi}(\boldsymbol{q})
$$

$$
\tilde{\chi}(\boldsymbol{q})=\int \mathrm{d} \varrho e^{-i \boldsymbol{q} \cdot \boldsymbol{p}} \tilde{\chi}(\varrho)
$$

$\varepsilon(\boldsymbol{q})$ being the dielectric constant, where the factorization has been used

$$
\left\langle\chi\left(\boldsymbol{r}, \boldsymbol{r}^{\prime \prime}\right) U\left(\boldsymbol{r}^{\prime \prime}\right)\right\rangle \cong \tilde{\chi}\left(\boldsymbol{r}-\boldsymbol{r}^{\prime \prime}\right)\left\langle U\left(\boldsymbol{r}^{\prime \prime}\right)\right\rangle
$$

which appears legitimate for a weak $U\left(\boldsymbol{r}^{\prime \prime}\right)$.

So far only the R.P.A. approximation has been used, essentially in Eq. (2.3), so that the equations are still "RPA" exact. As a next step we assume that the Green functions in (2.6) are those obtained neglecting the Coulomb interaction, as the leading term in (2.6) is already proportional to the interaction itself, and then we will use the following approximate decoupling for the ensemble average

$$
\begin{aligned}
& \left\langle G\left(\boldsymbol{r}^{\prime}, \boldsymbol{r}^{\prime \prime} ; \varepsilon\right) G\left(\boldsymbol{r}^{\prime \prime}, \boldsymbol{r}^{\prime} ; \varepsilon\right)\right\rangle \\
& \cong \gamma\left(\boldsymbol{r}^{\prime}-\boldsymbol{r}^{\prime \prime}\right) \tilde{G}\left(\boldsymbol{r}^{\prime \prime}-\boldsymbol{r}^{\prime} ; \varepsilon\right)
\end{aligned}
$$

where $\gamma\left(\boldsymbol{r}^{\prime}-\boldsymbol{r}^{\prime \prime}\right)$ satisfies the integral equation

$\int \mathrm{d} \boldsymbol{r}^{\prime \prime} \gamma\left(\boldsymbol{r}^{\prime}-\boldsymbol{r}^{\prime \prime}\right) \tilde{G}\left(\boldsymbol{r}^{\prime \prime}-\boldsymbol{r}^{\prime} ; \varepsilon\right)=-\frac{\partial}{\partial \varepsilon} \tilde{G}\left(\boldsymbol{r}^{\prime}-\boldsymbol{r}^{\prime} ; \varepsilon\right)$

which was introduced succesfully elsewhere ${ }^{7}$ and will be discussed in Appendix A. We anticipate however a result to be used in the following, i. e. that (2.10) becomes exact at long wavelenghts, that is when $\boldsymbol{q} \rightarrow 0$. In order to exploit this property fully we rewrite Eq. (2.9), by the help of (2.6), 
(2.10) and (2.11) in the form

$$
\begin{aligned}
\tilde{\chi}(\boldsymbol{q})= & \frac{4 \pi Z^{2} e^{2}}{q^{2}} \int_{-\infty}^{+\infty} \mathrm{d} \varepsilon f(\varepsilon) \operatorname{Im} \\
& \cdot \int \frac{\mathrm{d} \boldsymbol{k}}{(2 \pi)^{3}} \gamma(\boldsymbol{k}-\boldsymbol{q}) \tilde{G}(\boldsymbol{k})
\end{aligned}
$$

$\gamma(\boldsymbol{k})$ and $\tilde{G}(\boldsymbol{k})$ being the Fourier transform of $\gamma(\boldsymbol{r})$ and $\tilde{G}(\boldsymbol{r})$ (when not explicitly needed the energy index $\varepsilon$ will be neglected as an argument); where:

$$
\gamma(\boldsymbol{k})=-\frac{\partial}{\partial \varepsilon} \ln \tilde{G}(\boldsymbol{k})
$$

follows from 2.11). On substituting this result in (2.12) we find:

$$
\begin{aligned}
\tilde{\chi}(\boldsymbol{q})= & -\frac{4 \pi Z^{2} e^{2}}{q^{2}} \int_{-\infty}^{+\infty} \mathrm{d} \varepsilon f(\varepsilon) \operatorname{Im} \int \frac{\mathrm{d} \boldsymbol{k}}{(2 \pi)^{3}} \\
& \cdot \tilde{G}(\boldsymbol{k}) \tilde{G}(\boldsymbol{k}-\boldsymbol{q})\left(1-\frac{\partial}{\partial \varepsilon} \Sigma_{\boldsymbol{k}}(\varepsilon)\right)
\end{aligned}
$$

where use has been made of the conventional form $\tilde{G}(\boldsymbol{k})=\left[\varepsilon-\varepsilon_{\boldsymbol{k}}-\Sigma_{\boldsymbol{k}}(\varepsilon)\right]^{-1}, \varepsilon_{\boldsymbol{k}}=\left(\hbar^{2} / 2 m\right) k^{2}$ being the kinetic energy and $\Sigma_{\boldsymbol{k}}(\varepsilon)$ the self-energy, which in the approximation used incorporates only the effects of the random potential.

On using this form again it is easily found that

$$
\begin{gathered}
G(\boldsymbol{k}) \tilde{G}(\boldsymbol{k}-\boldsymbol{q})=\frac{1}{\varepsilon_{\boldsymbol{k}}+\Sigma_{\boldsymbol{k}}(\varepsilon)-\varepsilon_{\boldsymbol{k}-\boldsymbol{q}}-\sum_{\boldsymbol{k}-\boldsymbol{q}}(\varepsilon)} \\
\cdot\left[\frac{1}{\varepsilon-\varepsilon_{\boldsymbol{k}}-\Sigma_{\boldsymbol{k}}(\varepsilon)}-\frac{1}{\varepsilon-\varepsilon_{\boldsymbol{k}-\boldsymbol{q}}-\Sigma_{\boldsymbol{k}-\boldsymbol{q}}(\varepsilon)}\right]
\end{gathered}
$$

which in the limit of $\boldsymbol{q} \rightarrow 0$ can be approximated by replacing the denominator by $E_{\boldsymbol{k}}-E_{\boldsymbol{k}-\boldsymbol{q}}$, where $E_{k}$ is a solution of the equation

$$
E_{\boldsymbol{k}}-\varepsilon_{\boldsymbol{k}}-\Sigma_{1 \boldsymbol{k}}\left(E_{\boldsymbol{k}}\right)=0
$$

where $\Sigma_{1 \boldsymbol{k}}(\varepsilon)$ is the real part of $\Sigma_{\boldsymbol{k}}(\varepsilon)$, which is made possible by the additional fact that the Green functions in the bracket in (2.15) are strongly peaked at those energies satisfying (2.16).

Thus as long as we retain the $\boldsymbol{q} \rightarrow 0$ behaviour the equations written down so far are still asymptotically exact, within the RPA framework of course.

Equation (2.14) can then be brought into the final form:

$$
\tilde{\chi}(\boldsymbol{q})=-\frac{4 \pi Z^{2} e^{2}}{q^{2}} \int \frac{\mathrm{d} \boldsymbol{k}}{(2 \pi)^{3}} \frac{F_{\boldsymbol{k}}-F_{\boldsymbol{k}-\boldsymbol{q}}}{E_{\boldsymbol{k}}-E_{\boldsymbol{k}-\boldsymbol{q}}}
$$

with

$$
F_{\boldsymbol{k}}=\int_{-\infty}^{+\infty} \mathrm{d} \varepsilon f(\varepsilon) \operatorname{Im} \tilde{G}(\boldsymbol{k})\left(1-\frac{\partial}{\partial \varepsilon} \Sigma_{\boldsymbol{k}}(\varepsilon)\right)
$$

the full expression for the dielectric constant being then obtained by means of (2.8) .

So far the result (2.17) is still completely general as we have not specified $\Sigma_{\boldsymbol{k}}(\varepsilon)$ nor, for a given self-energy, we have studied any particular energy range of interest. As can be verified for example on taking $\Sigma_{\boldsymbol{k}}(\varepsilon) \rightarrow 0$ Eq. $(2.18)$ becomes $F_{\boldsymbol{k}}=f\left(\varepsilon_{\boldsymbol{k}}\right)$ namely the Fermi-Dirac distribution, and then Eq. (2.17) reduces to Lindhard's expression ${ }^{5}$, a check for the correctness of the decoupling procedure.

Turning now to the case of non vanishing $\Sigma_{\boldsymbol{k}}(\varepsilon)$, let us suppose that the temperature (T) is about zero, so that the integral in (2.18) can approximately be taken over the effective integration range $\left(-\infty \ldots \varepsilon_{\mathrm{F}}\right)$ due to $f(\varepsilon)$, where $\varepsilon_{\mathrm{F}}$ is the Fermi energy. Furthermore it will be assumed that $\varepsilon_{\mathrm{F}}$ lies well inside the region of localized states such that at $T \cong 0$ the occupancy of extended states is negligible and that the following equations can be used in $\left(-\infty \ldots \varepsilon_{\mathrm{F}}\right)$

$$
\begin{gathered}
\Sigma_{2 \boldsymbol{k}}(\varepsilon)=0 \\
1-\frac{\partial}{\partial \varepsilon} \Sigma_{1 \boldsymbol{k}}(\varepsilon)<0
\end{gathered}
$$

$\Sigma_{2 \boldsymbol{k}}(\varepsilon)$ being the imaginary part of the self-energy, valid whenever a state of energy $\varepsilon$ is localized ${ }^{8-10}$.

On splitting $\Sigma_{\boldsymbol{k}}(\varepsilon)$ into its real and imaginary parts and using (2.19) and (2.20) Eq. (2.18) becomes

$$
F_{\boldsymbol{k}} \cong-f\left(E_{\boldsymbol{k}}\right), \quad T \cong 0,
$$

so that finally $\varepsilon(\boldsymbol{q})$ acquires the form:

$$
\varepsilon(\boldsymbol{q})=1+\frac{4 \pi Z^{2} e^{2}}{q^{2}} \int \frac{\mathrm{d} \boldsymbol{k}}{(2 \pi)^{3}} \frac{f\left(E_{\boldsymbol{k}}\right)-f\left(E_{\boldsymbol{k}-\boldsymbol{q}}\right)}{E_{\boldsymbol{k}}-E_{\boldsymbol{k}-\boldsymbol{q}}}
$$

where the change in sign of the second term on the right hand side as compared to Lindhard's result is to be noted.

This is a main point as it implies the vanishing of $\varepsilon(\boldsymbol{q})$ at a certain $\boldsymbol{q}_{0}$; in fact in the limit of small $\boldsymbol{q}$, in $(2.22)$, consistently with the approximation used, we get

$$
\begin{aligned}
\varepsilon(\boldsymbol{q}) & =1+\frac{4 \pi Z^{2} e^{2}}{q^{2}} \int \frac{\mathrm{d} \boldsymbol{k}}{(2 \pi)^{3}} \\
& \cdot \frac{\partial f\left(E_{\boldsymbol{k}}\right)}{\partial E_{\boldsymbol{k}}} \frac{\nabla E_{\boldsymbol{k}} \cdot \boldsymbol{q}}{\nabla E_{\boldsymbol{k}} \cdot \boldsymbol{q}+\nabla_{2} E_{\boldsymbol{k}} q^{2} / 2}
\end{aligned}
$$

$+($ higher order derivatives of $f$ )

which vanishes when

$$
\begin{aligned}
q^{2}= & 4 \pi Z^{2} e^{2} \int \frac{\mathrm{d} \boldsymbol{k}}{(2 \pi)^{3}} \\
& \left(-\frac{\partial f\left(E_{\boldsymbol{k}}\right)}{\partial E_{\boldsymbol{k}}}\right) \frac{\nabla E_{\boldsymbol{k}} \cdot \boldsymbol{q}}{\nabla E_{\boldsymbol{k}} \cdot \boldsymbol{q}+\nabla_{2} E_{\boldsymbol{k}} q^{2}}
\end{aligned}
$$


owing to the fact that $\partial f\left(E_{\boldsymbol{k}}\right) / \partial E_{\boldsymbol{k}}<0$ everywhere at $T \cong 0$. The physical interpretation of the dielectric instability will be discussed in connection with metals later. In the following a simple example will be given of the calculation of $q_{0}$.

\section{An Example}

A simple example will be presented now to elucidate the most important steps of the calculations and assumptions needed to get (2.24). We then consider a system of charged particles interacting with a random collection of scatterers, whose concentration is $N$, fixed in space; each spatial configuration can be thought to be weighted by a probability function of the spatial coordinates of the scatterers, which we assume statistically independent such that the self energy of the charges is given by

$$
\Sigma_{\boldsymbol{k}}(\varepsilon)=N t_{\boldsymbol{k} \boldsymbol{k}}(\varepsilon)
$$

where $t_{\boldsymbol{k} \boldsymbol{k}}(\varepsilon)$ are the matrix elements of the $t$ matrix appropriate to a single scatterer. As we shall see later Eq. (3.1) can be used in metals to describe the interaction between an electron and a nucleus surrounded by the core. As a next assumption not strictly necessary, apart for a simplification of the mathematics, we will assume that $t_{\boldsymbol{k} \boldsymbol{k}}(\varepsilon)$ is a slowly varying function of $\boldsymbol{k}$ such that one can use the on the energy shell approximation

$$
t_{\boldsymbol{k} \boldsymbol{k}}(\varepsilon) \cong t_{\boldsymbol{k}_{0} \boldsymbol{k}_{0}}(\varepsilon)=t(\varepsilon)
$$

where $k_{0}{ }^{2}=\left(2 \mathrm{~m} / \hbar^{2}\right) \varepsilon$ : again this assumption maintains its validity in the metals to be discussed later.

With this simplified model, localized states are obtained at energies $\varepsilon$ satisfying the equations:

$$
\begin{gathered}
t_{2}(\varepsilon)=0, \\
\varepsilon-\varepsilon_{\boldsymbol{k}}-N t_{1}(\varepsilon) \geqq 0
\end{gathered}
$$

where $t_{1}(\varepsilon)$ and $t_{2}(\varepsilon)$ are the real and imaginary parts of $t(\varepsilon)$. On using the optical theorem ${ }^{11}$ :

$$
t_{2}(\varepsilon)=-(\hbar / \sqrt{2 m}) \sqrt{\varepsilon} \sigma(\varepsilon)
$$

where $\sigma(\varepsilon)$ is the total cross section of a scatterer, we see from (3.3) that $\varepsilon<0$. The existence of negative solutions of Eq. (3.4) can be studied by means of the following dispersion relation ${ }^{12}$ :

$N t_{1}(\varepsilon)=\tilde{V}+\frac{\hbar N}{\sqrt{2 m} \pi} \int_{0}^{\infty} \frac{\sqrt{\varepsilon^{\prime}} \sigma\left(\varepsilon^{\prime}\right)}{\varepsilon-\varepsilon^{\prime}} \mathrm{d} \varepsilon^{\prime}+\mathcal{T}(\varepsilon)$

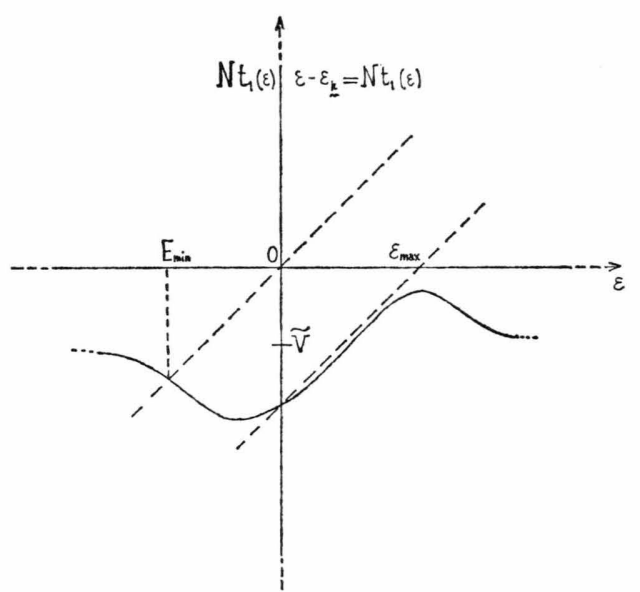

Fig. 1. Graphical solution of Eq. (3.4), obtained by plotting $\varepsilon-\varepsilon \boldsymbol{k}$ (straight dashed lines) and $N t_{1}(\varepsilon)$ from (3.6) (full line) as functions of $\varepsilon$ for a weak $\tilde{V}$ case.

where $\tilde{V}$ is the average potential (in space) and $\mathcal{T}(\varepsilon)$ a contribution from bound states, which we shall neglect on assuming that they are, if any, energetically far from the region of interest here. For a weak, attractive $\tilde{V}$ the graphical solution of Eq. (3.4) is given in Fig. 1 which shows negative solutions to exist for $\varepsilon_{\boldsymbol{k}} \leqq \varepsilon_{\max }$. They are in $\left(E_{\min } \ldots 0\right)$, where $E_{\min }$ is given by $E_{\min } \cong N t_{1}(0)$. From (3.6) it follows now that $N t_{1}(0)=\tilde{V}$ $+0\left(m^{-1 / 2}\right)$ so that for particles of large mass one can use $E_{\min } \cong \tilde{V}$.

Thus localized states of a system of fermions of large mass subjected to a weak, attractive random potential of $N$ scatterers per unite volume are in the interval $(\tilde{V} \ldots 0)$. The actual solutions $E_{\boldsymbol{k}}$ are not easily obtained in closed form and we shall assume that approximately

$$
E_{\boldsymbol{k}}=\varepsilon_{\boldsymbol{k}}+\tilde{V} .
$$

It is to be noted that this equation is assumed for the correct numerical value of $E_{\boldsymbol{k}}$ and is not to be taken as the correct functional form which in fact would violate Equation $(2.20)$. To verify that this latter is also satisfied in $(\tilde{V} \ldots 0)$ one should start from (3.6) and differentiate under the integral sign, but this can be done on general grounds ${ }^{10}$. On substituting now Eq. (3.7) in (2.24) it is found that

$$
\begin{aligned}
q^{2}= & 4 \pi Z^{2} e^{2} 2 \frac{2 \pi}{8 \pi^{3}} \int \mathrm{d} k k^{2} \frac{\partial f\left(E_{\boldsymbol{k}}\right)}{\partial \varepsilon_{\boldsymbol{k}}} \\
& \cdot\left[2-\frac{q}{2 k} \ln \left|\frac{1+q / 2 k}{1-q / 2 k}\right|\right]
\end{aligned}
$$


where $\mathrm{d} k=2 k^{2} \sin \vartheta \mathrm{d} k \mathrm{~d} \vartheta \mathrm{d} \varphi$ has been used in polar coordinates and an extra factor 2 takes account of the spin degeneracy. On retaining now the most divergent terms in the integrand in (3.8) it then follows that

$$
q_{0}{ }^{2} / 4 k_{\mathrm{F}}{ }^{2}=1+\exp \left(8 \pi \hbar^{2} k_{\mathrm{F}} / 2 m Z^{2} e^{2}\right)
$$

where

$$
k_{\mathrm{F}}=\frac{\sqrt{2 m}}{\hbar}\left(\varepsilon_{\mathrm{F}}-\tilde{V}\right)^{1 / 2}
$$

is the Fermi momentum. The order of magnitude of the exp term in (3.9) can be estimated for example by using $k_{\mathrm{F}} \cong\left(3 \pi^{2} N_{\mathrm{a}}\right)^{1 / 3}, N_{\mathrm{a}}$ being the number of particles per unite volume, volid for free fermions ${ }^{13}$; we then geht $\sim \exp \left(4 \times 10^{-3}\right)$ from $m=10^{3} \times$ electron mass, $Z=1$ and $N_{\mathrm{a}} \cong 10^{22} \mathrm{~cm}^{-3}$; this means that Eq. (3.9) in practical calculations becomes

$$
q_{0}^{2} / 4 k_{\mathrm{F}}^{2} \cong 2
$$

as a good approximation.

\section{The Dynamical Case}

The previous results for the static dielectric constant are extended to the dynamical case without difficulty. As a simple rule which reminds of the frequency dependence of the dielectric constant in Lindhard's case we can replace the denominator in (2.22) by $E_{\boldsymbol{k}}-E_{\boldsymbol{k}-\boldsymbol{q}}-\omega$, where $\omega$ is the frequency of the applied $U(\boldsymbol{r})$ now space-time dependent. In the limit $\omega \rightarrow 0$ then we can derive a dielectric instability by expanding the denominator in $(2.22)$ in powers of $\omega$; the first non vanishing contribution is thus seen to arise from $\omega^{2}$ terms and thus

is found, where

$$
m \omega=\hbar k_{\mathrm{F}} p, \quad q \rightarrow 0
$$

$$
k_{\mathrm{F}}{ }^{2}=\frac{\int_{0}^{\infty} \mathrm{d} k k^{2} \frac{\partial f\left(E_{\boldsymbol{k}}\right)}{\partial \varepsilon_{\boldsymbol{k}}}}{\int_{0}^{\infty} \mathrm{d} k \frac{\partial f\left(E_{\boldsymbol{k}}\right)}{\partial \varepsilon_{\boldsymbol{k}}}}
$$

defines again the Fermi momentum, according to (3.10), due to the fact that

$$
\partial f\left(E_{\boldsymbol{k}}\right) / \partial \varepsilon_{\boldsymbol{k}} \cong \delta\left[\varepsilon_{\boldsymbol{k}}-\left(\varepsilon_{\mathrm{F}}-\tilde{V}\right)\right]
$$

at low temperatures. This longitudinal acustic mode of vanishing $\omega$ as $q \rightarrow 0$ is the dynamical counterpart of the static mode given by (3.11) and has a simple interpretation in metals; the group velocity of the mode coincides with the Fermi velocity of the particles. It is to be noted that the static mode has likewise vanishing $\omega$ and is strictly obtained only retaining the $q^{2}$ term in the denominator of (2.24).

\section{Application to Simple Metals}

We now want to show that in simple metals, where the interaction between the electrons and nuclei can approximately be neglected ${ }^{14}$, the conditions of the previous paragraphs are recovered and the nuclei undergo an instability, which can be taken as responsible for the existence of the crystal lattice.

Let us then consider a system of nuclei, of charge $Z e$, and electrons, of charge $e$, neutral in the sense that if $N_{\mathrm{a}}$ is the nuclei concentration $Z N_{\mathrm{a}}$ is the electron concentration. In alkali metals for example it is useful to think of the nuclei plus core, where the core is built up by the closed shells, as the $Z e$ charged system, $Z$ being the valence. When the adiabatic theorem holds ${ }^{15}$ i.e. provided the ratio $m_{\mathrm{e}} / m$ between the electron mass $m_{\mathrm{e}}$ and the nucleus plus core mass $m$ is small, the nuclear motion obeys the effective Schrödinger's equation:

$$
\begin{aligned}
& {\left[-\frac{\hbar^{2}}{2 m} \sum_{i} \frac{\partial^{2}}{\partial \boldsymbol{R}_{i}{ }^{2}}+\frac{1}{2} \sum_{i \neq j} \frac{Z^{2} e^{2}}{\left|\boldsymbol{R}_{i}-\boldsymbol{R}^{j}\right|}+E\left(\left\{\boldsymbol{R}_{i}\right\}\right)\right]} \\
& \cdot \Phi\left(\left\{\boldsymbol{R}_{i}\right\}\right)=\mathcal{E} \Phi\left(\left\{\boldsymbol{R}_{i}\right\}\right)
\end{aligned}
$$

where the nuclei are thought as interacting with electrons and with themselves, and where $\left\{\boldsymbol{R}_{i}\right\}$, with $\boldsymbol{R}_{i}$ the position of the $i$-th nucleus, denotes the generic configuration. $E\left(\left\{\boldsymbol{R}_{i}\right\}\right)$ is given by ${ }^{15}$ :

$$
\begin{aligned}
E\left(\left\{\boldsymbol{R}_{i}\right\}\right)= & \int \mathrm{d}\left\{\boldsymbol{r}_{j}\right\} \psi^{*} \\
& \cdot\left(\left\{\boldsymbol{r}_{j}\right\} ;\left\{\boldsymbol{R}_{i}\right\}\right) \mathcal{H} \psi\left(\left\{\boldsymbol{r}_{j}\right\} ;\left\{\boldsymbol{R}_{i}\right\}\right)
\end{aligned}
$$

where $\mathcal{H}$ and $\psi$ are the hamiltonian and wavefunction for the electrons in a frozen configuration $\left\{\boldsymbol{R}_{i}\right\}$, i. e.:

$$
\begin{aligned}
\mathcal{H}= & -\frac{\hbar^{2}}{2 m_{\mathrm{e}}} \sum_{\alpha} \frac{\partial^{2}}{\partial \boldsymbol{r}_{\alpha}{ }^{2}}+\frac{1}{2} \sum_{\alpha \neq \beta} \frac{e^{2}}{\left|\boldsymbol{r}_{\alpha}-\boldsymbol{r}_{\beta}\right|} \\
& +\sum_{\alpha i} V\left(\boldsymbol{r}_{\alpha}-\boldsymbol{R}_{i}\right)
\end{aligned}
$$

$V\left(\boldsymbol{r}_{\mathrm{a}}-R_{i}\right)$ being the electron-nucleus interaction, so that $E\left(\left\{\boldsymbol{R}_{i}\right\}\right)$ is the eigenvalue which corresponds to a given $\psi$ in a fixed configuration $\left\{\boldsymbol{R}_{i}\right\}$, not necessarily the periodic configuration. We can then regard (5.1) as Schrödinger's equation in a random potential, the random coordinates being those of the electrons, the probability density being

$$
\left|\psi\left(\left\{\boldsymbol{r}_{i}\right\} ;\left\{\boldsymbol{R}_{j}\right\}\right)\right|^{2}
$$


and we can furthermore regard it is a lowest order approximation, i.e. an average potential approximation, which follows from (5.2), the potential being $\mathcal{H}$ as from $(5.3)^{16}$. This interpretation becomes very simple only if $\psi$ does not depend on $\left\{\boldsymbol{R}_{i}\right\}$ in such a way that a well defined probability function, independent of the nuclear motion itself, can be assigned to the electron coordinates, but in principle this is not required.

If we consider the simplest metals however it is satisfied; in fact then $\psi$ will be an antisymmetric Slater determinant built up from plane waves and hence independent of $\left\{\boldsymbol{R}_{i}\right\}$ such that $E\left(\left\{\boldsymbol{R}_{i}\right\}\right)$ can be splitted into two contributions, one coming from the first two terms in (4.3) which depend only on the electron coordinates, identical for all metals, and another containing the electron-nucleus interaction. By means of a suitable energy scale choice we can neglect the former while we can interpret the latter as the average potential on each nucleus generated by the electrons, randomly distributed in space as $\mid \psi^{2}$ of each electron is proportional to $1 / \Omega$, where $\Omega$ is the volume of the system. Thus we recover the conditions of paragraph 3 so that we derive that the metals considered here, provided the nuclei plus core are fermions, their Fermi level being inside localized states at $T \cong 0$, are unstable against formation of a lattice, whose spacing is $a_{0}=2 \pi / q_{0}$ where $q_{0}$ is given by (3.9), and there exists a collective dynamical mode around the lattice whose frequency is given by (4.1), which can be interpreted as a phonon dispersion relation in the acoustic domain. Testing this interpretation requires the calculation of $k_{\mathrm{F}}$ which determines both the lattice spacing and the frequency of vibration. To do that we rewrite Eq. (3.10) as

$$
\varepsilon_{\mathrm{F}}=\left(\hbar^{2} / 2 m\right) k_{\mathrm{F}}^{2}+V
$$

which is an energy conservation relation, $k_{\mathrm{F}} / m$ playing the role of a velocity, so that we can apply the virial theorem ${ }^{17}$

$$
\tilde{V}=-2\left(\hbar^{2} / 2 m\right) k_{\mathrm{F}}^{2}
$$

on assuming for example that the electron-nucleus potential is of the Coulomb type. In effect a screened Coulomb potential will be assumed for convergence reasons, but actually by final results will be independent of the screening radius. We thus have

$$
\tilde{V}=-N_{\mathrm{a}} Z 4 \pi\left(Z e^{2} / \lambda^{2}\right)
$$

where $\lambda$ is the inverse of the screening radius, which can be eliminated in favour of the density of states $N\left(\varepsilon_{\mathrm{F}}^{\prime}\right)$ of the electrons at the Fermi level according to ${ }^{18}$ :

$$
4 \pi\left(e^{2} / \lambda^{2}\right)=1 / N\left(\varepsilon_{\mathrm{F}}^{\prime}\right)
$$

thereby showing the actual independence from $\lambda$. By means of (3.10) we then find

$$
U_{\mathrm{F}}=\hbar \frac{k_{\mathrm{F}}}{m}=\left(\frac{N_{\mathrm{a}} Z^{2}}{m N\left(\varepsilon_{\mathrm{F}}^{\prime}\right)}\right)^{1 / 2}
$$

which is a common result for the velocity of sound in alkali metals ${ }^{19}$. It is convenient to note that $\varepsilon_{\mathrm{F}}{ }^{\prime}$ is the Fermi energy of the electrons now, which arises from the fact that the screening parameter $\lambda$ is determined by the electrons in this framework. One thus finds that acoustic phonons can be viewed as collective modes generated by the localization induced by the random potential of the electrons, plus Coulomb interactions.

In a similar way, on using (3.11) and (5.8) the lattice spacing is obtained as

$$
a_{0}=1.26 / N_{\mathrm{a}}{ }^{1 / 3} Z^{5 / 6}
$$

where $N\left(\varepsilon_{\mathrm{F}}{ }^{\prime}\right)=\frac{3}{2} N_{\mathrm{a}} Z / \varepsilon_{\mathrm{F}}{ }^{\prime}$ and

$$
\varepsilon_{\mathrm{F}}^{\prime}=\left(\hbar^{2} / 2 m_{\mathrm{e}}\right)\left(3 \pi^{2} N_{\mathrm{a}} Z\right)^{2 / 3}
$$

are used as appropriate to the free Fermi electron gas ${ }^{20}$.

In Table 1 we report the atomic masses of the alkali metals as well as other quantities of interest. The spins of the nuclei, with the exception of the rare isotopes ${ }^{6} \mathrm{Li}$ and ${ }^{40} \mathrm{~K}$, are odd so that we are dealing with fermions as required. Explicit calculations for $a_{0}$ are based on the $N_{\mathrm{a}}$ values of this table and the results are summarized in Table 1 where they are compared with the experimental values. The agreement is almost perfect.

In the framework used here $q_{0}$ is the wavevector of the collective mode so that $a_{0}$ should be interpreted as the wavelength, that is the spatial unit which repeats periodically in the mode; thus we find the obvious interpretation of $a_{0}$ as the lenght of the unit cell which is the spatial repeating unit. We cannot deduce from the calculation the number of atoms in the cell but we can infer that the cell must be cubic as only one solution exists to the dielectric instability; one notes, interestingly enough, that all alkali metals have cubic cells, although the number of atoms inside the cell is greater than one owing to the b.c.c. structure. 


\begin{tabular}{|c|c|c|c|c|c|c|c|}
\hline \multicolumn{2}{|c|}{ Element-Z- } & \multirow{2}{*}{$\begin{array}{c}\begin{array}{c}\text { Atomic mass } \\
\text { (average) }\end{array} \\
6.939\end{array}$} & \multirow{2}{*}{$\begin{array}{l}\begin{array}{l}\text { Crystal } \\
\text { structure }\end{array} \\
\text { b.c.c. }\end{array}$} & \multicolumn{2}{|l|}{$a$ in $\AA$} & \multirow{2}{*}{$\begin{array}{l}\begin{array}{l}\text { Density in } \\
\mathrm{g} \mathrm{cm}^{-3}\end{array} \\
0.542^{78 \mathrm{~K}}\end{array}$} & \multirow{2}{*}{$\begin{array}{l}\mathrm{Na} \times 10^{24} \\
{\text { in } \mathrm{cm}^{-3}}^{-3} \\
0.047\end{array}$} \\
\hline $\mathrm{Li}$ & 1 & & & (3.459) & $3.491^{78} \mathrm{~K}$ & & \\
\hline $\mathrm{Na}$ & 1 & 22.989 & b.c.c. & $(4.208)$ & $4.225^{5 \mathrm{~K}}$ & $1.013^{5 \mathrm{~K}}$ & 0.026 \\
\hline $\mathrm{K}$ & 1 & 39.102 & b.c.c. & (5.174) & $5.225^{5 \mathrm{~K}}$ & $0.910^{5 \mathrm{~K}}$ & 0.014 \\
\hline $\mathrm{Rb}$ & 1 & 85.470 & b.c.c. & (5.586) & $5.585^{5 \mathrm{~K}}$ & $1.6295 \mathrm{~K}$ & 0.011 \\
\hline Cs & 1 & 132.910 & b.c.c. & $(5.983)$ & $6.045^{5 \mathrm{~K}}$ & $1.9975 \mathrm{~K}$ & 0.009 \\
\hline
\end{tabular}

Table 1. Parameters of alkali metals taken from Ref. ${ }^{14} \cdot a$ is the length of the (cubic) unit cell. The superscripts on the right indicate the temperature each value refers to, the numbers in parentheses indicate the theoretical values $a_{0}$ of this paper.

\begin{tabular}{|c|c|c|c|c|c|c|c|c|}
\hline \multicolumn{2}{|c|}{ Element- $Z$ - } & \multirow{2}{*}{$\begin{array}{c}\begin{array}{c}\text { Atomic mass } \\
\text { (average) }\end{array} \\
9.012\end{array}$} & \multirow{2}{*}{$\begin{array}{l}\begin{array}{l}\text { Crystal } \\
\text { structure }\end{array} \\
\text { h.c.p. }\end{array}$} & \multirow{2}{*}{\multicolumn{2}{|c|}{$\begin{array}{l}a \text { in } \AA \\
\begin{array}{r}(1.421) \\
c=3.27 \\
c=39\end{array}\end{array}$}} & \multirow{2}{*}{$\begin{array}{l}a / a_{0} \\
1.60\end{array}$} & \multirow{2}{*}{$\begin{array}{l}\begin{array}{l}\text { Density in } \\
\mathrm{g} \mathrm{cm}^{-3}\end{array} \\
1.82\end{array}$} & \multirow{2}{*}{$\begin{array}{l}\mathrm{Na} \times 10^{24} \\
\text { in } \mathrm{cm}^{-3}\end{array}$} \\
\hline $\mathrm{Be}$ & 2 & & & & & & & \\
\hline $\mathrm{Ba}$ & 2 & 137.34 & b.c.c. & $(2.776)$ & 5.02 & 1.81 & 3.59 & 0.255 \\
\hline $\mathrm{Al}$ & 3 & 26.98 & f.c.c. & (1.277) & 4.05 & 3.17 & 2.70 & 0.395 \\
\hline In & 3 & 114.82 & tetr. & $\begin{array}{r}(1.479) \\
c=\end{array}$ & $\begin{array}{r}3.25 \\
=4.95\end{array}$ & 2.20 & 7.29 & 0.341 \\
\hline $\mathrm{Zn}$ & 2 & 65.37 & h.c.p. & $\begin{array}{r}(1.744) \\
c=\end{array}$ & $\begin{array}{r}2.66 \\
=4.95\end{array}$ & 1.53 & 7.13 & 0.406 \\
\hline $\mathrm{Pb}$ & 4 & 207.19 & f.c.c. & (1.225) & 4.95 & 4.04 & 11.34 & 0.324 \\
\hline
\end{tabular}

Table 2. Parameters of non alkali metals which can be treated as fermions, referring to room temperature. The numbers in parentheses are the theoretical $a_{0}$ values of this paper. The ratio $a / a_{0}$ is also reported. $c$ is the additional length for non cubic cells.
If other so called simple metals are considered and only those having uneven spin, according to the criterium given above, are selected, the results, even if neglecting the c length for non cubic structures, are much worse as follows from Table 2 , and one notes an increasing disagreement for increasing $Z$, a result certainly due to the fact that the electronnucleus interaction is no longer negligible as far as the wavefunctions of the electrons are concerned. These metals are thus less "simple" than the alkali metals.

\section{Conclusion}

The stringent evidence of the agreement between experimental and theoretical $a_{0}$ values and velocities of sound in this simplified model calculation indicates that the nuclear localization, as due to the random potential of the electrons, is a basis for understanding the origin of the crystal lattice as well as lattice vibrations in simple metals.

We have indicated how it works in this simple case but in principle no objection exists against an improvement such as taking the electron-nucleus coupling into account in a more satisfactory way. The theory is also restricted to fermions, the reason being an expected different behaviour for bosons. The Pauli principle forbids in fact that more than two particles be in the same localized state so that an increasing number of such states will be filled on increasing the number of particles. Thus an increasing portion of space will be occupied even in the presence of a relatively weak RPA field.

Bosons on the other hand would condensate in a single state at $T \sim 0$ thus avoiding the occupation of increasing volume. It is doubtful if an RPA field description can be accepted in this case, the effective Coulomb interaction being much stronger. It appears that statistics discriminates strongly the two kind of particles although spin doesn't appear explicitly.

We note that Eq. (2.14) can be interpreted diagramatically as a low-order loop in the expansion of the proper polarization part $\tilde{\chi}(\boldsymbol{q})$ normalized by the vertex $1-\partial \Sigma_{\boldsymbol{k}} / \partial \varepsilon$; so in effect higher order diagrams are included in a self-consistent way, coming from the self-energy renormalization used in the propagator lines: it is the vertex that is responsible for the instability, which would disappear within a bare framework.

Equations (2.19) and (2.20) on the other hand, which the instability relies upon, are taken from a transport formulation valid for localized states and are related to the vanishing of the static conductivity as a function of energy at absolute zero, when the energy belongs to a localized state [see Eqs. (5.3) to (5.4) of Ref. ${ }^{8}$ ] and Ref. ${ }^{10}$ for equivalence between the two. 
Some points which we didn't consider are the inclusion of exchange and correlation beyond the RPA and wave function effects due to core electrons. The first point, as far as exchange is concerned, appears of decisive importance as it is expected to produce spin arrangements and spin interactions which are absent in this zero-spin formulation and it is our purpose to continue investigating this point althought we believe that the crystallization picture will remain unchanged.

Correlation, on the other hand, appears difficult to examine but a posteriori the excellent agreement between calculated and measured values of the lattice spacing seems to exclude any appreciable effect.

Finally core electrons have not been considered as far as their wavefunctions are concerned because only valence electrons are expected to generate the random potential; there does not appear to be any other simple way to treat them and we are doubtful whether a formal general treatment including them from the start would give an appreciable improvement for the alkali metals: the size of the core in these metals is too small as compared to the volume available to valence electrons (see Reference ${ }^{14}$ ).

\section{Appendix A: \\ Gauge Invariance and Functional Consistency}

There are two requirements that the decoupling $(2.10)-(2.11)$ must satisfy, namely gauge invariance and functional consistency.

Gauge invariance is expressed by the following Ward's identity ${ }^{21}$ :

$$
\left\langle\hat{G}\left(\varepsilon_{1}\right) \hat{G}\left(\varepsilon_{2}\right)\right\rangle=-\frac{1}{\varepsilon_{1}-\varepsilon_{2}}\left\langle\hat{G}\left(\varepsilon_{1}\right)-\hat{G}\left(\varepsilon_{2}\right)\right\rangle
$$

to be satisfied by the Green function operator $\hat{G}(\varepsilon)$ $=\left(\varepsilon-\hat{p}^{2} / 2 m-\hat{V}\right)^{-1}$ the matrix elements of which in space representation are involved in (2.10), $\hat{p}^{2} / 2 m$ being the kinetic energy and $\hat{V}$ the random potential, to be taken as the fourth component of the tetra-potential describing an electromagnetic field. In particular when $\varepsilon_{1} \rightarrow \varepsilon_{2}$ (A.1) becomes

$$
\langle\hat{G}(\varepsilon) \hat{G} \varepsilon)\rangle=-\frac{\partial}{\partial \varepsilon}\langle\hat{G}(\varepsilon)\rangle
$$

and taking matrix elements in real space we get

$$
\begin{array}{r}
\int \mathrm{d} \boldsymbol{r}^{\prime \prime}\left\langle G\left(\boldsymbol{r}, \boldsymbol{r}^{\prime \prime} ; \varepsilon\right) G\left(\boldsymbol{r}^{\prime \prime}, \boldsymbol{r}^{\prime} ; \varepsilon\right)\right\rangle \\
=-\frac{\partial}{\partial \varepsilon}\left\langle G\left(\boldsymbol{r}, \boldsymbol{r}^{\prime} ; \varepsilon\right)\right\rangle
\end{array}
$$

an identity which is readily seen to be satisfied by the approximation (2.11) for $\gamma\left(\boldsymbol{r}-\boldsymbol{r}^{\prime}\right)$. This proves that gauge invariance is preserved by the decoupling.

The second point is less trivial as it is a requirement on the fact that the decoupling (2.10) must also preserve the correct functional form of an average of a product of Green functions, which is not guaranteed a priori by the choice $(2.11)$ for $\gamma(\boldsymbol{r})$. We can conveniently examine this point in Fourier space as in fact Eq. (2.10) becomes

$$
\begin{gathered}
\int \frac{\mathrm{d} \boldsymbol{k}}{(2 \pi)^{3}}\left\langle G_{\boldsymbol{k}^{\prime}, \boldsymbol{k}}(\varepsilon) G_{\boldsymbol{k}-\boldsymbol{q} \cdot \boldsymbol{k}^{\prime \prime \prime}}(\varepsilon)\right\rangle \\
=G\left(\boldsymbol{k}^{\prime}\right) G\left(\boldsymbol{k}^{\prime \prime}\right)\left(1-\frac{\partial}{\partial \varepsilon} \Sigma_{\boldsymbol{k}^{\prime \prime \prime}}(\varepsilon)\right) \delta_{\boldsymbol{k}^{\prime}-\boldsymbol{q}, \boldsymbol{k}^{\prime \prime \prime}}
\end{gathered}
$$

which will be simpler to examine.

We now expand the left hand side of (A.4) in powers of the potential according to a standard method and then verify that the right hand side is consistent, in a functional sense, with the exact expansion. The whole expansion however need not to be carried on to all orders and a few low order terms suffice to prove the consistency.

Let's then expand the Green functions on the left hand side of (A.4) according to (we neglect $\varepsilon$ again for simplicity) :

$$
\begin{aligned}
G_{\boldsymbol{k}^{\prime}, \boldsymbol{k}}= & G_{\boldsymbol{k}}^{0} \delta_{\boldsymbol{k}, \boldsymbol{k}^{\prime}}+G_{\boldsymbol{k}^{\prime}}^{0} V_{\boldsymbol{k}^{\prime}, \boldsymbol{k}} G_{\boldsymbol{k}}^{0} \\
& +\int \frac{\mathrm{d} \boldsymbol{k}^{\prime \prime}}{(2 \pi)^{3}} G_{\boldsymbol{k}^{\prime}}^{0} V_{\boldsymbol{k}^{\prime}, \boldsymbol{k}^{\prime \prime}} G_{\boldsymbol{k}^{\prime \prime}}^{0} V_{\boldsymbol{k}^{\prime \prime}, \boldsymbol{k}} G_{\boldsymbol{k}}^{0}+\ldots
\end{aligned}
$$

where $G_{\boldsymbol{k}}^{0}$ is the unperturbed Green function and $V_{\boldsymbol{k}^{\prime}, \boldsymbol{k}}$ the matrix elements of the random potential which are given by:

$$
\begin{aligned}
G_{\boldsymbol{k}}^{0} & =\left(\varepsilon-\hbar^{2} k^{2} / 2 m-i \eta\right)^{-1} ; \quad \eta=0_{+}, \\
V_{\boldsymbol{k}^{\prime}, \boldsymbol{k}} & =\sum_{i} \exp \left\{-i\left(\boldsymbol{k}^{\prime}-\boldsymbol{k}\right) \cdot \boldsymbol{R}_{i}\right\} v_{\boldsymbol{k}^{\prime}, \boldsymbol{k}}
\end{aligned}
$$

where we assume that the randomness of the potential is due to the random variables $\boldsymbol{R}_{i}$, to be considered as positions from where the potential acting on each particle of the system is developed. This particular choice was used in par. 5 , by identifying $\boldsymbol{R}_{i}$ as the random position of the electrons interacting with the nuclei in metals.

We have then

$$
\begin{aligned}
& \int \frac{\mathrm{d} \boldsymbol{k}}{(2 \pi)^{3}}\left\langle G_{\boldsymbol{k}^{\prime}, \boldsymbol{k}} G_{\boldsymbol{k}-\boldsymbol{q}, \boldsymbol{k}^{\prime \prime \prime}}\right\rangle=G_{\boldsymbol{k}}^{0} G_{\boldsymbol{k}^{\prime \prime \prime}}^{0} \delta_{\boldsymbol{k}^{\prime}-\boldsymbol{q}, \boldsymbol{k}^{\prime \prime \prime}} \\
& +N_{\mathrm{a}} Z G_{\boldsymbol{k}^{\prime}}^{0} G_{\boldsymbol{k}^{\prime \prime \prime}}^{0} v_{\boldsymbol{k}^{\prime \prime \prime}, \boldsymbol{k}^{\prime \prime \prime}} G_{\boldsymbol{k}^{\prime \prime \prime}}^{0} \delta_{\boldsymbol{k}^{\prime}-\boldsymbol{q}, \boldsymbol{k}^{\prime \prime \prime}} \\
& +N_{\mathrm{a}} Z G_{\boldsymbol{k}^{\prime}}^{0} v_{\boldsymbol{k}^{\prime}, \boldsymbol{k}^{\prime}} G_{\mathbf{k}^{\prime}}^{0} G_{\boldsymbol{k}^{\prime \prime \prime}}^{0} \delta_{\boldsymbol{k}^{\prime}-\boldsymbol{q}, \boldsymbol{k}^{\prime \prime \prime}}+\ldots
\end{aligned}
$$


$N_{\mathrm{a}} Z$ being the density of electrons in the metal, which developes a structure where each terms is of the form

$$
\alpha\left(\boldsymbol{k}^{\prime}, \boldsymbol{k}, \boldsymbol{q}, \boldsymbol{k}^{\prime \prime \prime}\right) \delta_{\mathbf{k}^{\prime}-\boldsymbol{q}, \boldsymbol{k}^{\prime \prime \prime}}
$$

so that one easily verifies the functional consistency of (A.4), which is of course an approximation from the numerical point of view but is thus exact from the functional point of view. We can, as a particular case, of relevance in this paper, verify that as $q \rightarrow 0$,

1 P. W. Anderson, Phys. Rev. 109, 1492 [1958].

2 D. J. Thouless, Phys. Rep. 13, 93 [1974].

3 N. F. Mott, Phil. Mag. 34, 643 [1976].

4 A. L. Efros, J. Phys. C 9, 2021 [1976].

5 J. M. Ziman, "Principles of the Theory of Solids", p. 126, Cambridge Univ. Press, London 1965.

6 D. N. Zubarev, Sov. Phys. Usp. 3, 320 [1960].

7 V. Dallacasa, J. Phys. C 8, 114 [1974].

8 V. Dallacasa, Sol. St. Comm. 18, 777 [1976].

9 D. Mattis and F. Yonezawa, Phys. Rev. Lett. 31, 828 [1973].

10 V. Dallacasa, Lett. Nuovo Cim. 14, 324 [1975].

11 L. Landau and E. Lifchitz, "Mécanique Quantique", p. 557, Editions Mir, Moscow 1966.

12 Same as Ref. ${ }^{11}$, p. 583.

13 Same as Ref. ${ }^{5}$, p. 69.

14 C. Kittel, "Introduction to Solid State Physics", p. 239, John Wiley \& Sons, New York 1971.
(A.4) becomes exact also numerically as in fact the left hand side becomes then

$$
-\frac{\partial}{\partial \varepsilon} \tilde{G}_{\boldsymbol{k}^{\prime}, \boldsymbol{k}}
$$

where

$$
\tilde{G}_{\boldsymbol{k}^{\prime}, \boldsymbol{k}}=G(\boldsymbol{k}) \delta_{\boldsymbol{k}^{\prime}, \boldsymbol{k}}
$$

so that, on using again the standard form for $G(\boldsymbol{k})$, this is verified to coincide with the right hand side of (A.4).

15 Same as Ref. ${ }^{5}$, p. 169.

${ }^{16}$ For an exaustive description of a random potential one is referred to Reference ${ }^{2}$.

17 H. Goldstein, "Classical Mechanics", 2nd ed., p. 64, Addison-Wesley, Tokyo 1950.

18 See Ref. ${ }^{5}$, p. 130.

19 See for example Ref. ${ }^{14}$, p. 712.

20 These formulas can be verified for example in Ref. ${ }^{14}$, p. 247 and 249. The direct calculation from (3.11) leads rigorously to the wavelength of the static mode with respect to the electrons at rest because we have neglected their kinetic energy and (5.9) follows on changing to the laboratory frame and taking the ratio of the velocity of the electrons to the velovity of the nuclei $V_{\text {elec }} / V_{\text {nucl }} \cong$ $\mathrm{m} / \mathrm{m}_{\mathrm{e}}$ into account.

21 B. Velicky, Phys. Rev. 184, 614 [1969]. 